\title{
Incidence, Trends and Epidemiology of Cancers in North West of Iran
}

\author{
Atefeh Zahedi ${ }^{1}$, Hosein Rafiemanesh ${ }^{2}$, Mostafa Enayatrad ${ }^{2}$, Mahshid Ghoncheh ${ }^{3}$, \\ Hamid Salehiniya ${ }^{4,5 *}$
}

\begin{abstract}
Background: Cancer is a leading cause of death throughout the world. Increasing life expectancy and aging population are important factors for increasing cancer incidences in developing countries. National programs are essential for prevention and control of cancer in any society. This study aimed to investigate cancer epidemiology and trends in the province of Hamadan, located in Northwest Iran. Materials and Methods: This analytical study was carried out based on cancer registry data from 2004 to 2009 in the province of Hamadan, analyzed using STATA (version 12) software for descriptive tests and Join point 4.1.1.1 software for analytical tests. Results: There were 7,767 registered cases of cancer during the 6 years studied. Of the total cases registered, $59.1 \%(4,592$ cases) involved men and $40.9 \%$ (3,175 cases) occurred in women. Age-standardized incidence rates (ASR) increased from 72.9 to 132.0 in males and 48.2 to 115.0 in females during the 6 years of the study (p<0.001). The most common cancers were skin, stomach, breast, bladder, and leukemia. In women, teh most common were breast, skin, stomach, colorectal, and leukemia, in that order, and in men skin, stomach, bladder, leukemia, and prostate cancers. Conclusions: The cancer incidence is greater in men that women in this region but with increasing trends in both sexes. Planning regarding education in prevention of exposure to risk factors and control strategies is required to decrease the incident cases. Screening programs for common cancers in older age groups might be helpful to reduce the disease impact.
\end{abstract}

Keywords: Cancer - trend - epidemiology - Hamadan - Iran

Asian Pac J Cancer Prev, 16 (16), 7189-7193

\section{Introduction}

Cancer is defined as rapid development of abnormal cells that can spread to other organs (Neda Amoori, 2014; Ghoncheh et al., 2015; Keyghobadi, 2015). It is one of the leading causes of death in developed and developing countries (Bryere, 2014; Keyghobadi, 2015; Razi et al., 2015). In the past decades, unhealthy lifestyle was an important risk factor in western countries and currently the factor increased cancer in developing countries(Jemal, 2010). Furthermore, increasing life expectancy and aging population are other important factors for enhancing cancer incidence in these countries (Thun, 2010; Keyghobadi, 2015) so that the burden of disease has increased more than twice (Harirchi, 2011).

According to epidemiological and demographic transition of population and changed patterns of mortality in Iran, cancer is the third cause of death after cardiovascular diseases and accidents (Kolahdoozan et al., 2010). Therefore, national programs are essential for prevention and control of cancer in any society. Cancer registry is considered as a main tool for management and control of the disease (Goya, 2007). In population based cancer registries, there is data collected from cancer incidence and mortality from the disease obtained from a target group to describe epidemiology of cancer in a defined region (Zanetti and Chen, 2013), plan cancer control strategies, conduct, and evaluate cancer prevention and control effects by scientific research. Cancer statistics is widely used in scientific studies, clinical trials, and making decisions on cancer prevention and control (Chen, 2013).

The primary purposes of cancer control programs is early detection, effective treatment, and palliative care programs, in which target population is not limited to certain age, sex, ethnicity, and race (Goya, 2007). Since knowing disease status in any society is necessary to prevention planning and cancer control, especially in high

${ }^{1}$ Department of Biostatistics and Epidemiology, Faculty of Health, Shahid Sadoughi University of Medical Sciences, Yazd, ${ }^{2}$ Department of Epidemiology and Biostatistics, School of Public Health, Shahid Beheshti University of Medical Sciences, ${ }^{4}$ Minimally Invasive Surgery Research Center, Iran University of Medical Sciences, ${ }^{5}$ Department of Epidemiology and Biostatistics, School of Public Health, Tehran University of Medical Sciences, Tehran, ${ }^{3}$ Department of Epidemiology and Biostatistics, School of Public Health, Hamadan University of Medical Sciences, Hamadan, Iran*For correspondence: alesaleh70@yahoo.com 
prevalence areas, this study aimed to investigate cancer epidemiology and trend in the province of Hamadan, located in North West of Iran..

\section{Materials and Methods}

This was an analytical study carried out based on cancer registry data and the disease management center of health ministry from 2004 to 2009 in the province of Hamadan. Data used in this study was obtained from a national registry of cancer (NCR), and Disease Control and Prevention (CDC) of ministry of Health and Medical Education in Iran for 2004 to 2009 in Iran, there are 30 provinces and 41 Medical Universities. Deputy for health of each university is responsible for health issues of the population and all health activities are managed by these deputies. All deputies for health have been included in the NCR. Registrar would apply the national registration software which was developed by CDC. For pathologic centers, without software, the cancer records were gathered manually. The Cancer Office of CDC should provide techniques and funding supports. The data are transmitted every 3 months, by electronic file and also hard copy of 'Cancer Registry Data Collection Form'; this form is comprised of three parts: part I, regarding patient's identity characteristics in addition to the name of biopsy-taker physician, name of hospital, location of which the biopsy is taken, clinical diagnosis and date of biopsy sent to histological laboratory and demographic information of the patients includes race and residence. Part II includes the most important findings of patient's clinical history. Part III includes preclinical findings. The information includes primary location of tumor, date of cancer diagnosis, morphology and histology and its behavior and diagnosis method. Physicians fill the form of clinical data and the official personnel fill the identity and demographic information. Quality control has been coordinated in five main areas by Cancer Office of CDC: (i) regarding completeness of coverage; (ii) completeness of details; (iii) accuracy of data; (iv) accuracy of reports; (v) accuracy of interpretation and (vi) repeated cases are deleted from national data. Surveillance of pathology is based on the cancer record in several selected provinces to compare it with the present pathology cancer record for a general and complete evaluation and also for the accuracy of the collected data. IARC software provides a way to identify inaccuracies in data coding. Accordance of The International Classification of Diseases for Oncology (ICD-OC: topography with ICD-OM: morphology) is done manually and also by considering age and sex groups (pathology file of fatal error has been revised by the Scientific Society of Pathology of Iran and also by two masters in pathology).

Method for deleting repeated cases: for the lack of any classified National Identification Numbers, the process for deleting the repeated cases was completed by a manual review of the record. After editing data of each province and considering in mind that for deleting of repeated cases, similar cases should also be the same as morphology, topography, identity and demographic information; deletion of the repeated cases would be done separately in each province and finally in all over country by experienced manual reviewers.

Data extracted were studied based on the number of cases and standardized incidence rates for box sexes and sex ratio. Standardized incidence rates were directly calculated based on the world standard population. Data was analyzed using STATA (version 12) software. To calculate the annual percentage change (APC) and trend, Join point 4.1.1.1 software was applied. The significance level was considered as 0.05 . Common cancers were defined as the number of reported cases and standardized incidence rates.

\section{Results}

In this study, there were 7,767 registered cases of cancer during 6 years studied. Of the total cases registered, $59.1 \%$ (4,592 cases) were men and $40.9 \%$ (3,175 cases) occurred in women. The sex ratio, according to the number of cancer cases, (male to female) for all cancers was 1.45. The highest number of cancer cases ( 704 cases) was seen in women in 2009 and the greatest number of cancer cases ( 1,067 cases) was observed in 2008 in men. The total number of cancer cases was 781 cases in 2004 and the number was increased to 1,635 cases in 2009 , as changes in cases were more than twice and APC was equal to 109.3.

The Age-Standardized Rate (ASR) was increased

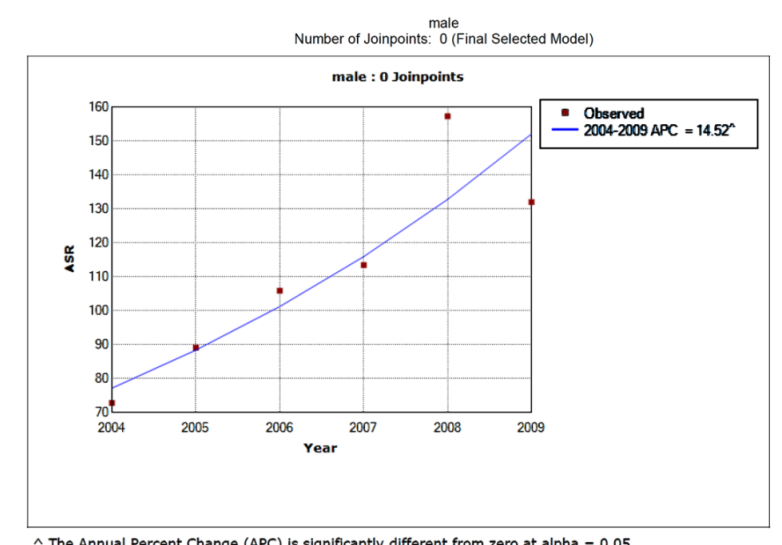

Figure 1. Join Point Analysis of Incidence Cancer in Men in Hamedan, Iran, 2004 - 2009. ASR (W) - agestandardized rate per 100000 (using world standard population)

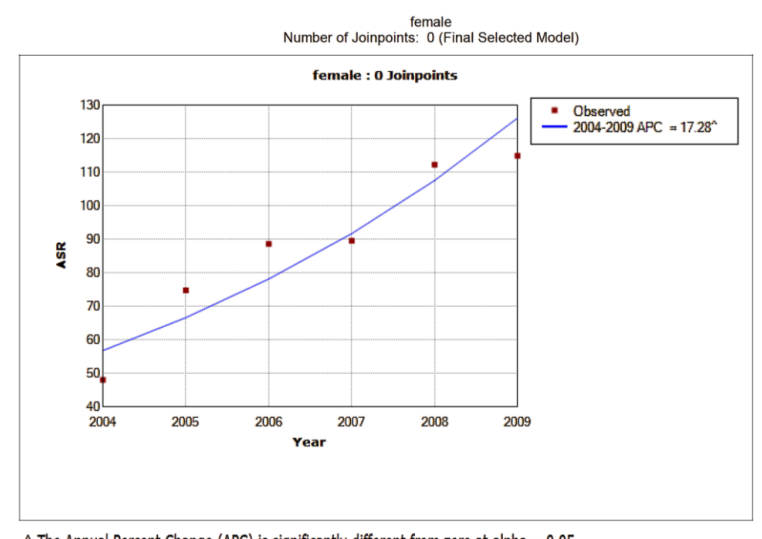

^ The Annual Percent Change (APC) is significantly different from zero at alpha $=0.05$

Figure 2. Join Point Analysis of Incidence Cancer in Women in Hamedan, Iran, 2004 - 2009. ASR (W) - agestandardized rate per 100000 (using world standard population) 
Table 1. Join Point Analyses of Cancers Incidence in Hamedan, Iran, 2004 - 2009, with the Estimated Annual Percent Change (APC) and 95\% Confidence Intervals

\begin{tabular}{|c|c|c|c|c|c|c|c|c|c|}
\hline & \multicolumn{3}{|c|}{ Trend 1} & \multicolumn{3}{|c|}{ Trend 2} & \multicolumn{3}{|c|}{ Full Range } \\
\hline & years & APC & $95 \% \mathrm{CI}$ & years & APC & $95 \% \mathrm{CI}$ & years & AAPC & $95 \% \mathrm{CI}$ \\
\hline Male & 2004- 2007 & $19.1^{*}$ & 6.8 to 32.8 & 2007- 2009 & 7.2 & -13.8 to 33.3 & 2004- 2009 & $14.2 *$ & 12.3 to 16.1 \\
\hline Female & 2006- 2009 & 33.1 & -69.8 to 486.3 & 2006- 2009 & 8.8 & -48.1 to 128.5 & 2004- 2009 & $18.0^{*}$ & 5.2 to 32.2 \\
\hline
\end{tabular}

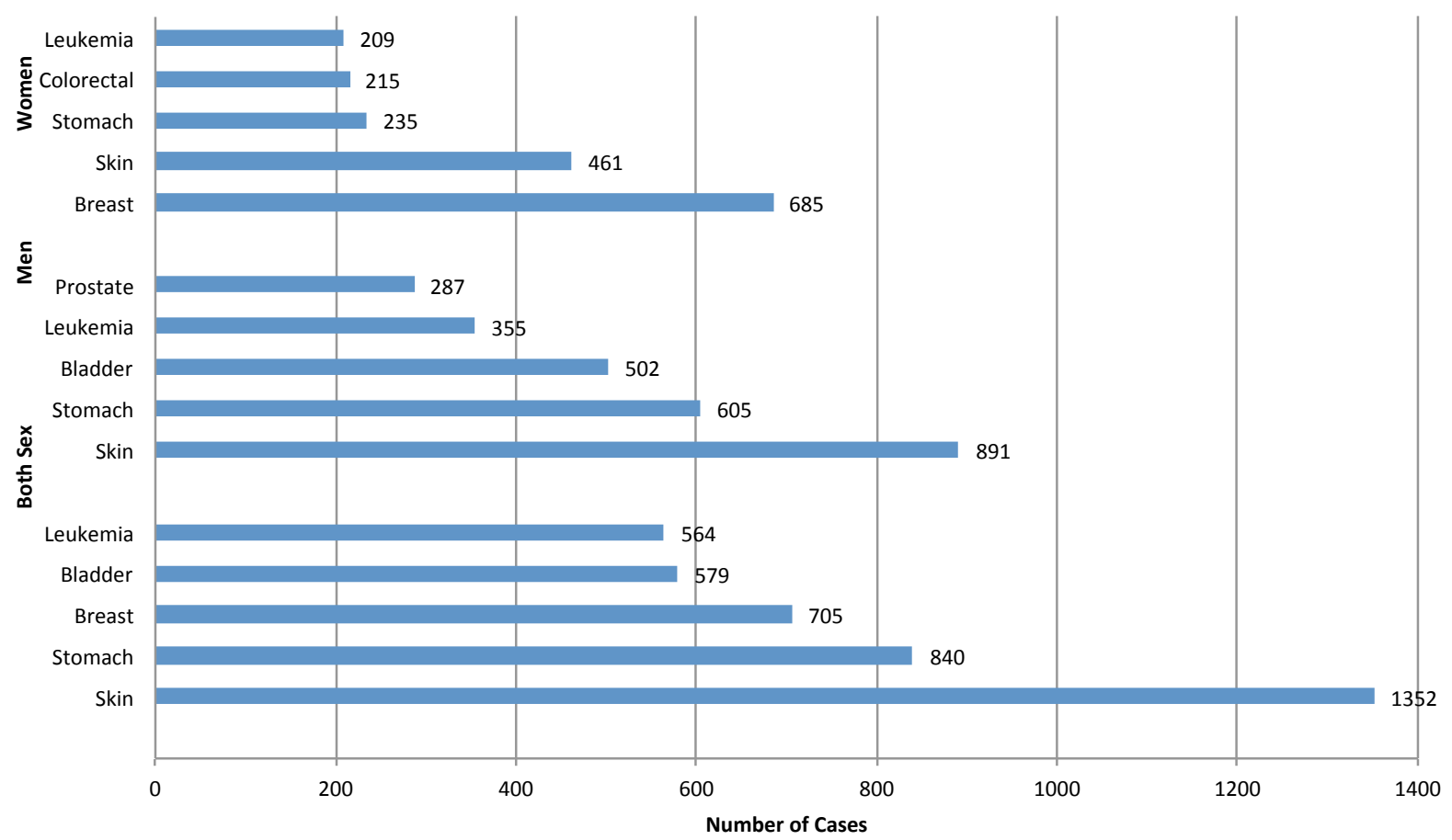

Figure 3. Five Common Cancer and Number of Cases for by Sex and Both Sex in Hamedan, Iran (2004 - 2009)

from 72.88 to 132.03 in males between 2004 and 2009 and the increasing trend was significant according to mean percentage changes of 14.52 (CI: 6-23.8, $\mathrm{p}<0.001$ ) (Figure1). In females, the rate was increased from 48.17 to 114.98 during 6 years of the study so that the increasing trend was significant considering mean percentage changes of 17.28 (CI: 7.6-27.9, p<0 .001) (Figure 2). The cancer incidence trend is shown in Table 1 by three-year periods (2004-2006, 2007-2009).

Mean age-standardized incidence rate was 111.81 per 100,000 people in males and 88.12 per 100,000 people in females. Overall, the most common cancers in both sexes during six years studied were skin, stomach, breast, bladder, and leukemia, respectively. The most common cancers were breast, skin, stomach, colorectal, and leukemia in women during 6 years, respectively, but in men skin stomach, bladder, leukemia, and prostate cancers, respectively (Figure 3).

\section{Discussion}

In this study, common cancers were investigated by sex in the province of Hamadan during 2004 and 2009. The largest number of cases occurred in men $(59.1 \%$ vs.40.9\%). In the province of eastern Azerbaijan, located in North West of Iran, the greatest number of cases was seen in men (63.01\% vs.336.67) between 2007 and 2001(Ferlay J, 2013). As well, the rate was more in males than females in Europe (53\% vs.47\%)(Fateh M, 2013). In our study, mean percentage of 14.52 in men and 17.28 in women indicated significant increases in trend of disease $(\mathrm{p}<0.001)$. Another study in Iran showed that APC was $12.4 \%$ (Siegel, 2014) while in United States (U.S), the incidence rate has slightly decreased in men ( $6 \%$ per year) but it has been constant in women during 2006 to 2011 (Mousavi et al., 2009). The incidence of all cancers in developed countries than in developing countries in both men and women is nearly double while the rate of death from cancer in developed countries, only $21 \%$ in males and $2 \%$ higher in women. This inequality in patterns of incidence and mortality between developed regions and underdeveloped regional differences in the prevalence and distribution of risk factors, diagnostic methods and availability and use of health care services reflects (Mousavi, 2009).

Highest increase in cancer cases was seen in men and women in 2008 , can be attributed to changes in the cancer registry system in the country because reports on cancer cases was made based on pathology reports, but reports of cancer cases were done as population based from 2008. According to improving the registry system, the probability of not reporting cases was very low. Therefore, an increased peak was observed in men and women in 2008. Another study in Iran also showed that the sex ratio (male to female) was 14.5 while the ratio for all cancers was 1/1.2 in the country (Mousavi, 2009). 
ASR was increased from 72.88 to 132.03 in males and from 48.17 to 114.98 between 2004 and 2009, which is related to lifestyle changes such as increasing consumption of fast foods, fatty foods, and sedentary lifestyle. Mean age-standardized incidence rate was 111.81 per 100,000 people in males and 88.12 per 100,000 people in females, which was lower than ASR in Korea (345.1 in males and 300.9 in females per 100,000 people) and Serbia (307.2 in males and 256.4 in males) (Jung et al., 2012b; Mihajlovic et al., 2013). The most common cancer was skin in men (ASR=22.44), but a study in Shahrood, a city in Iran, showed skin was the second most common cancer in men (Mousavi et al., 2009). While it was not among the fifth common cancers in Korea, China, Europe, Serbia (Mousavi et al., 2009; Jung et al., 2012a; Ferlay, 2013). In other words, Iranian men do not protection actions against sun's ultraviolet radiation. Hence, notification is required.

In 2008989,600 new cases and 738,000 deaths due to stomach cancer occurred in the world. More than $70 \%$ of new cases and deaths are in developing countries. The rate of gastric cancer in men is about twice that of women. The highest incidence of gastric cancer in East Asia, Eastern Europe and South America and lowest in North America and many parts of Africa. The geographical differences reflect differences in dietary patterns, particularly in European countries, the prevalence of $\mathrm{H}$. pylori infection Ferlay and Steliarova-Foucher, 2010). Stomach cancer was the second most common cancer in men in Hamadan $(\mathrm{ASR}=14.88)$, but the cancer is the first common cancer in Iranian men $(\mathrm{ASR}=15)$ (Ferlay and Steliarova-Foucher, 2010). Another study in Iran showed that stomach is the first common cancer in males (Mousavi, 2009), similar to Korea (Ferlay et al., 2013). It was the fifth cancer in Europe (Xie et al., 2012). Bladder cancer (ASR=12.73) was the third common cancer in men in Hamadan and the forth in men in Europe and Serbia (ASR=16.2) (Mousavi et al., 2009; Jung et al., 2012a). Also the incidence of stomach cancer is increasing in Iran, so further epidemiological studies into the etiology and early detection are essential (Almasi et al., 2015).

In this study, breast cancer was the first common cancer in women (ASR=17.98), which was lower than the country (ASR=24) (Mousavi et al., 2009; Enayatrad, 2015). In other studies, the cancer was the first in women (Mousavi, 2009; Razavi, 2009; Jung, 2012b; Siegel, 2014; Enayatrad M, 2015). The overall incidence of breast cancer in Western countries, Northern Europe, Australia, New Zealand and North America, the average in South America and North Africa and in sub-Saharan Africa and Asia is low. It was much lower in Iranian females than developed countries. This may be due to lifestyle and early detection facilities. In Iran, according to the high cost of diagnostic procedures, including mammography, and unavailability for everybody, women over 40 years do not regularly benefit from the diagnostic methods, which used only in emergency cases. Therefore, the factor can affect cancer statistics.

In our study, the second common cancer was skin in women (ASR=13.5) but it was not considered important cancers in females (Mousavi et al., 2009; Jung et al., 2012a; Ferlay et al., 2013; Razi et al., 2015). Given Iranian women's coverage and their lower exposure, it is expected that skin cancer incidence rate is low but I Hamadan the cancer was the second common cancer in females. As a result, it is necessary to conduct education programs for skin care against ultraviolet radiation.

In this study, the third common cancer was stomach in women (ASR=6.88). It is the fourth in Iranian females (ASR=6.65), similar to Korea (24.7) (Jung et al., 2012b).

Colorectal cancer is the third most common cancer in men and the second most common cancer in women, more than 2.1 million new cases and 608,700 deaths due to colorectal cancer occurred in 2008. The highest incidence rates in Australia, New Zealand, Europe and North America, while the lowest is in Africa and South and Central Asia. Colorectal cancer was the fourth common cancer in females in Hamadan (ASR=6.02), lower than the country (ASR=8.17). The fifth common cancer in women studied was leukemia (ASR=5.47). The cancer was not among five common cancers in all women (Mousavi et al., 2009; Razavi et al., 2009; Jung et al., 2012b; Ferlay et al., 2013).

The differences between incidence rates and the type of common cancers in this study compared with other studies can be due to racial and environmental differences, and lifestyle. It is necessary to carry out other studies on identifying effective risk factors, prevention and control of cancers.

In conclusion, our finding revealed that the cancer incidence was more in men that women in this region and associated with increasing trend in both sexes. Planning regarding education in prevention of exposure to risk factors and control strategies is required to decrease the incidence cases. Screening program for common cancers in older age groups can be helpful.

\section{References}

Almasi Z, Rafiemanesh H, Salehiniya H (2015). Epidemiology characteristics and trends of incidence and morphology of stomach cancer in Iran. Asian Pac J Cancer Prev, 16, 2757-61.

Bryere J DO, Bouvier V, Colonna M, Guizard A-V, Troussard $X$, et al (2014). Socioeconomic environment and cancer incidence: a French population-based study in Normandy. BMC Cancer, 14, 87.

Chen W ZR, Zhang S, Zhao P, Li G, Wu L, et al (2013). The incidences and mortalities of major cancers in China, 2009. Chinese J Cancer, 32, 106-12.

Enayatrad M SH (2015). An investigation of changing patterns in breast cancer incidence trends among Iranian women. Quarterly J Sabzevar University of Medical Sciences, 22, 27-35.

Fateh M EM ( 2013). Cancer incidence and trend analysis in Shahroud, Iran, 2000-2010. Iranian J Cancer Prev, 6, 85-94.

Ferlay J, Steliarova-Foucher E (2010). Estimates of cancer incidence and mortality in Europe in 2008. European $J$ Cancer, 46, 765-81.

Ferlay J S-FE, Lortet-Tieulent J, Rosso S, et al ( 2013). Cancer incidence and mortality patterns in Europe: Estimates for 40 countries in 2012. European J Cancer, 49, 1374-403.

Ghoncheh M, Mohammadian-Hafshejani A, Salehiniya H (2015). Incidence and mortality of breast cancer and their relationship to development in asia. Asian Pac J Cancer Prev, 16, 6081-7. 
Goya M, (2007). Iranian annual cancer registration report 2005/2006. ministry of health and medical education, health deputy. center for disease control and prevention 2007. [In Persian]. Tehran, Iran.

Harirchi I KS, Karbakhsh M, Chegini N, et al (2011). Twenty years of breast cancer in Iran: downstaging without a formal screening program. Annals Oncol, 22, 93-7.

Jemal A CM, DeSantis C, Ward EM (2010). Global patterns of cancer incidence and mortality rates and trends. Cancer Epidemiology Biomarkers \& Prevention, 19, 1893-907.

Jung K-W PS, Kong H-J, Won Y-J, et al (2012a). Cancer statistics in Korea: incidence, mortality, survival, and prevalence in 2009. Cancer Research and Treatment, 44, 11-24.

Jung K-W PS, Won Y-J, Kong H-J, et al (2012b). Prediction of cancer incidence and mortality in Korea, 2012. Cancer Research and Treatment, 44, 25-31.

Keyghobadi N RH, Mohammadian-Hafshejani A, Enayatrad M, Salehiniya H (2015). Epidemiology and trend of cancers in the province of Kerman: southeast of Iran. Asian Pac J Cancer Prev, 16, 1409-13.

Kolahdoozan S, Sadjadi A, Radmard AR, et al (2010). Five common cancers in Iran. Arch Iran Med, 13, 143-6.

Mihajlovic J PP, Miladinov-Mikov M, Zivkovic S, Postma MJ (2013). Cancer incidence and mortality in Serbia 1999-2009. BMC Cancer, 13, 1471-2407.

Mousavi SM GM, Ramazani R, Davanlou M, Hajsadeghi N, Seddighi Z (2009). Cancer incidence and mortality in Iran. Ann Oncol, 20, 556-63.

Neda Amoori MM, Maria Cheraghi (2014). Incidence of Cancers in Kuzestan Province of Iran: Trend from 2004 to 2008. Asian Pac J Cancer Prev, 15, 8345-49.

Razavi SE AH, Haghazali M, Nadali F, Ramazani R, Dabiri E, et al (2009). The most common cancers in Iranian women. Iranian J Public Health, 38, 109-12.

Razi S, Rafiemanesh H, Ghoncheh M, et al (2015). Changing trends of types of skin cancer in Iran. Asian Pac J Cancer Prev, 16, 4955-8.

Saeid Razi, Mostafa Enayatrad, abdollah MohammadianHafshejani, et al ( 2015). The Epidemiology of Skin Cancer and its Trend in Iran. Intern J Prev Med, 6, 65.

Siegel R MJ, Zou Z, Jemal A (2014). Cancer statistics, 2014. CA: A Cancer J Clinicians, 64, 9-29.

Thun MJ DJ, Center MM, Jemal A, Ward EM (2010). The global burden of cancer: priorities for prevention. Carcinogenesis, 31, 100-10.

Xie W-C CM-H, Mak K-C, Chan W-T, He M. (2012). Trends in the incidence of 15 common cancers in Hong Kong. Asian Pac J Cancer Prev, 13, 3911-6.

Zanetti R SI, Sacchetto L, Binder-Foucard F, Bordoni A, Coza $\mathrm{D}$, et al. Completeness and timeliness: Cancer registries could/should improve their performance. European Journal of Cancer. 